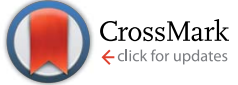

Cite this: RSC Adv., 2014, 4, 62413

Received 14th September 2014 Accepted 5th November 2014

DOI: 10.1039/c4ra10417j

www.rsc.org/advances

\section{New insight into the shape-controlled synthesis and microwave shielding properties of iron oxide covered with reduced graphene oxide $\uparrow$}

\author{
Ankit Gupta, ${ }^{\text {ab }}$ Avanish Pratap Singh, ${ }^{\mathrm{b}}$ Swati Varshney, ${ }^{a}$ Narayan Agrawal, ${ }^{a}$ \\ Pradeep Sambyal, ${ }^{b}$ Yashraj Pandey, ${ }^{a}$ Bhanu Pratap Singh, ${ }^{b}$ V. N. Singh, ${ }^{a}$ \\ Bipin Kumar Guptab and S. K. Dhawan*a
}

\begin{abstract}
We proposed various approaches for the shape-controlled synthesis of iron oxide-RGO composites to evaluate the effect of different morphologies on their microwave shielding properties. The nature of various ferrite structures (flakes, cubes and rods) covered by reduced graphene oxide multilayers has been investigated using X-ray diffraction, Raman spectroscopy, FT-IR, scanning electron microscopy, high-resolution transmission electron microscopy and $\mathrm{X}$-ray photoelectron spectroscopic techniques. The electromagnetic interference (EMI) shielding effectiveness of iron oxide of different shapes coated with reduced graphene oxide was investigated in the Ku band frequency range (12.4-18 GHz). The rod shaped iron oxide covered with reduced graphene oxide sheets demonstrates the highest shielding effectiveness value of $\sim 33.30 \mathrm{~dB}$ (>99.9\% attenuation) as compared to flake and cube shaped iron oxides due to the combined effect of magnetic losses (hysteresis, eddy current loss and effective anisotropy) and dielectric losses (space charge polarization, interfacial polarization, surface defects, multiple scattering, etc.). These innovative proposed structures and their obtained EMI shielding results deliver a new insight into the morphology dependent nature of iron oxides covered with RGO nanosheets and create new opportunities for next generation EMI materials.
\end{abstract}

\section{Introduction}

In recent times, there has been a remarkable increase in the demand for and use of electronic devices. A consequence of such devices is the generation of electromagnetic radiation, which is extremely harmful for human health and the environment. ${ }^{\mathbf{1 , 2}}$ Additionally, this electromagnetic radiation has adverse effects on many sensitive electronic devices that cause great degradation in their quality and disturb their normal operation. Therefore, it is necessary to develop materials for high performance electromagnetic interference (EMI) shielding. ${ }^{3,4}$ EMI shielding effectiveness depends on two mechanisms, i.e. reflection loss and absorption loss. Basically, reflection loss is the dominating factor for EMI shielding effectiveness. ${ }^{5}$ It results from the interaction between the conducting part of the composite and the electromagnetic field, whereas the absorption loss depends on the extent of the absorption of EM waves by the part of the composite material that has high magnetic

${ }^{a}$ Delhi Institute of Tool Engineering, New Delhi-110 020, India. E-mail: skdhawan@ mail.nplindia.ernet.in; Fax: +91-11-25726938; Tel: +91-11-45609401

${ }^{b}$ CSIR-National Physical Laboratory, Dr K. S. Krishnan Road, New Delhi-110 012, India

$\dagger$ Electronic supplementary information (ESI) available. See DOI: $10.1039 / \mathrm{c} 4 \mathrm{ra} 10417 \mathrm{j}$ permeability. ${ }^{4}$ Hence, the EMI SE of the material used as a barrier in the form of sheets or foam mainly depends on its dielectric properties, magnetic permeability, conductivity, frequency and flexibility. In the past decades, extensive research has been carried out to encapsulate different types of metal in graphene sheets to add additional functions to the matrix. The development of different ferrite nanostructures, such as rods, wires, cubes, flakes and spindles, has become a key emerging research field, due to their restricted dimensions, and controlled shape and size, which give rise to increased control over dispersion, hybridization, chemical, and thermal stability. Other specific electronic, magnetic and mechanical properties of such shape-controlled materials are superior to those of their bulk counterparts. ${ }^{6}$ In general, one dimensional (1D) nanostructures (rods, tubes and wires) are expected to show better electrical, magnetic and optical properties due to their anisotropic morphology. ${ }^{7}$ The synthesis of 1D, 2D and 3D (rod, flake and cube) structures provides an opportunity to study and compare the dependence of peculiar and intriguing chemical and physical properties on dimensionality and size confinement. There are several possibilities for further tailoring the EMI shielding properties of iron oxide by integrating it with materials that are highly conductive in nature, even if they have weaker magnetic properties. Graphene oxide has ultraconductive properties but lacks in magnetic properties, which 
are important for EM wave absorption, hence composites of ferromagnetic elements like $\mathrm{Ni}, \mathrm{Co}$, and $\mathrm{Fe}$ with graphene have attracted many researchers to use it in many applications like electromagnetic devices and drug delivery systems. ${ }^{8}$ Graphene oxide, being a two dimensional, planar layer of fused $\mathrm{sp}^{2}$ carbon bonds has emerged as an interesting material with many physical, electrical, magnetic and optical properties. ${ }^{9}$ In addition to the cost effectiveness of graphene oxide, its stable dispersion stability with many solvents, high thermal stability, ultra-high conductivity, high strength, high aspect ratio and layered structure make graphene oxide a strong alternative to CNTs. ${ }^{10}$ The presence of functional groups attached to the graphene sheets enables GO to form stronger bonds with different filler materials, to further aid the interfacial load transfer between GO and filler materials. Recently, chemically modified graphene based hybrid materials have proven to be fascinating materials for many technological applications..$^{\mathbf{1 1 1 - 1 3}}$ In light of this, an effort has been made to use the composites of reduced graphene oxide and ferrite nanoparticles for electromagnetic interference shielding applications. Among these, there have been many reports on the use of nanostructured iron oxideRGO composites for application in Li-ion battery electrodes and transistors. ${ }^{8}$ But, to the best of our knowledge, there have been very few reports on the use of the iron oxide-RGO matrix for EMI shielding applications. Moreover, there are no reports on the study of the EM waves absorption performance of the different nanostructures of ferrite particles and the compatibility of RGO coated iron oxide cubes, rods and flakes, and their further effects on the EMI shielding effectiveness. The present investigation deals with a novel approach to develop new composites based on different morphologies of iron oxide (rods/flakes/cubes of $\mathrm{Fe}_{2} \mathrm{O}_{3}$ ) covered with RGO nanosheets and their detailed characterization with their electromagnetic shielding properties.

\section{Experimental}

\subsection{Materials}

Natural graphite was used to synthesize reduced graphene oxide (rGO) and purchased from Loba Chemie India. Sulfuric acid, hydrazine hydrate and ammonia solution were procured from Rankem Limited. $\mathrm{FeSO}_{4} \cdot 7 \mathrm{H}_{2} \mathrm{O}, \mathrm{Fe}\left(\mathrm{NO}_{3}\right)_{3}, \mathrm{CH}_{3} \mathrm{COONa} \cdot 3 \mathrm{H}_{2} \mathrm{O}$, $\mathrm{Na}_{2} \mathrm{SO}_{3}, \mathrm{NaOH}$ and sodium dodecyl sulfate were procured from Merck, India. The other chemicals were of reagent grade and used as received.

\subsection{Synthesis of different structures of iron oxide}

Nanorods, microcubes and nanoflakes of iron oxide were synthesized via a simple sol-gel method in two steps (Fig. 1). ${ }^{\mathbf{1 4 , 1 5}}$ The first step involved a room temperature reaction. $A \mathrm{~g}$ of ' $\mathrm{X}$ ' was added to a solution of $B \mathrm{ml}$ distilled water and $C \mathrm{ml}$ ethanol to form a homogeneous solution. This was followed by the addition of $D \mathrm{~g}$ of ' $\mathrm{Y}$ '. After a few minutes of stirring, the color of the solution turned to a reddish brown. The reddish brown solution was vigorously stirred for $24 \mathrm{~h}$. A suspension was obtained after stirring and it was transferred to a Teflon lined autoclave, which was sealed and heated in a furnace at $T^{\circ} \mathrm{C}$ for $H \mathrm{~h}$. The autoclave used for the hydrothermal process had an inner diameter of $2.5 \mathrm{~cm}$, outer diameter of $4.2 \mathrm{~cm}$ and length of 10.2. The autoclave had a total volume of approximately $50 \mathrm{~cm}^{3}$ but only $70 \%$ of the autoclave $\left(35 \mathrm{~cm}^{3}\right)$ was filled with the suspension. Furthermore, the image of the actual set-up can be seen in the ESI (Fig. S1 $\dagger$ ). The hydrothermal process was carried out at $120^{\circ} \mathrm{C}, 160{ }^{\circ} \mathrm{C}$ and $180{ }^{\circ} \mathrm{C}$ for $\mathrm{Fe}_{2} \mathrm{O}_{3}$ rods, $\mathrm{Fe}_{2} \mathrm{O}_{3}$ cubes and $\mathrm{Fe}_{2} \mathrm{O}_{3}$ flakes, respectively. These different temperatures were responsible for controlling the nucleation of the various shaped structures of the hydroxide phase. In the second step of the synthesis, the precipitate was centrifuged at $10000 \mathrm{rpm}$ and washed with distilled water and ethanol several times. Then it was dried in a vacuum oven at $60{ }^{\circ} \mathrm{C}$ for $5 \mathrm{~h}$ and calcined at $650{ }^{\circ} \mathrm{C}$ for $3 \mathrm{~h}$ to obtain the oxide phase of the iron oxide with different shapes. The details of the above mentioned parameters and chemicals for the synthesis of rod, cube and flake shaped iron oxide are described in the table below (Table 1).

\subsection{Synthesis of reduced graphene oxide (RGO)}

For the synthesis of graphene oxide the modified Hummers method was used. Graphite powder ( $5 \mathrm{~g})$ and $\mathrm{NaNO}_{3}(5 \mathrm{~g})$ were mixed into concentrated $\mathrm{H}_{2} \mathrm{SO}_{4}(230 \mathrm{ml}) . \mathrm{KMnO}_{4}(40 \mathrm{~g})$ was added gradually to this solution with stirring and cooling, so that the temperature of the mixture was not allowed to exceed $20{ }^{\circ} \mathrm{C}$. The mixture was then stirred at $35{ }^{\circ} \mathrm{C}$ for $2 \mathrm{~h}$ and deionized water $(200 \mathrm{ml})$ was added. The reaction mixture was stirred for 1 hour after the addition of a large amount of deionized water $(300 \mathrm{ml})$ and $30 \% \mathrm{H}_{2} \mathrm{O}_{2}$ solution $(30 \mathrm{ml})$, causing violent effervescence and an increase in the temperature to $100{ }^{\circ} \mathrm{C}$, after which the colour of the suspension changed to bright yellow. The suspension was washed with $1: 10 \mathrm{HCl}$ solution $(100 \mathrm{ml})$ in order to remove the metal ions. The collected paste was dried at $60{ }^{\circ} \mathrm{C}$ in a vacuum oven. The resultant powder was dispersed in distilled water followed by ultrasonication for 4 hours. RGO was thereafter produced by reducing graphene oxide with hydrazine hydrate.

\subsection{Synthesis of RGO-iron oxide composites}

The RGO-iron oxide composites were prepared in the following steps: iron oxide nanorods and RGO with a weight ratio of $9: 1$ were added to an ethanol medium in the presence of sodium dodecyl sulfate (15\% of the total weight) as a cross-linking agent, followed by ultrasonication for $24 \mathrm{~h}$. Then the solution was dried at $110{ }^{\circ} \mathrm{C}$ for $8 \mathrm{~h}$ to obtain the iron oxide-RGO composites, which are abbreviated as RRods. Composites of RGO with iron oxide microcubes and nanoflakes were synthesized in a similar way and are abbreviated as RCubes and RFlakes, respectively.

\subsection{Materials characterization}

The particle size and the surface morphology of the RGO nanocomposites were examined using high-resolution transmission electron microscopy (HRTEM, Technai G20-stwin), using an accelerating voltage of $200 \mathrm{kV}$ with a point resolution of $1.44 \AA$ and a line resolution of $2.32 \AA$, and scanning electron 


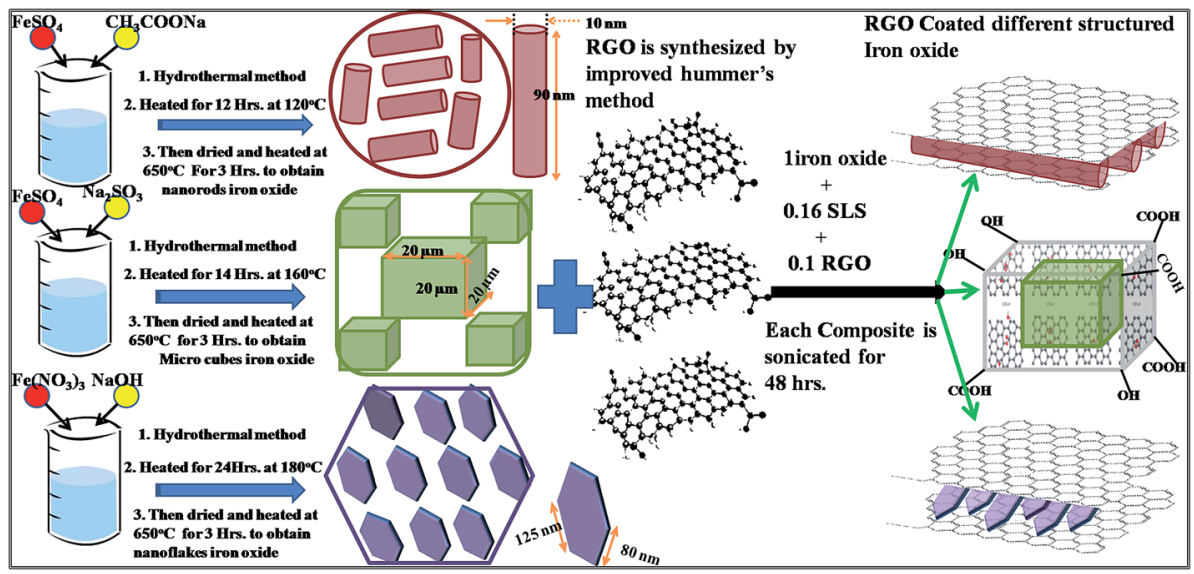

Fig. 1 Schematic representation of the shape-controlled synthesis of iron oxide covered with RGO.

microscopy (SEM LEO 440). The adsorption/desorption experiments were performed in a Perkin Elmer ultra-high vacuum XPS chamber (Model 1257) at a base pressure of $5 \times 10^{-10}$ torr. The XPS technique was used for the identification of the elemental composition and oxidation states. The chamber was equipped with a dual anode $\mathrm{Mg}-\mathrm{K} \alpha$ (energy $1253.6 \mathrm{eV}$ ) and Al-K $\alpha$ (energy $1486.6 \mathrm{eV}$ ) X-ray source and a high-resolution hemispherical energy analyzer for energy resolved electron detection. All binding energies (BE) were calibrated using the $\mathrm{BE}(284.6 \mathrm{eV})$ of $\mathrm{C} 1 \mathrm{~s}$, which gave BE values with an accuracy of $0.1 \mathrm{eV}$. The spectra were corrected for static charging by setting the $\mathrm{C} 1 \mathrm{~s}$ peak maximum at $285.28 \mathrm{eV}$.

The presence of ferrite particles in the composites was confirmed by X-ray diffraction (XRD) studies carried out on a D8 Advance X-ray diffractometer (Bruker) using $\mathrm{Cu} \mathrm{K} \alpha 1$ radiation $(\lambda=1.5406 \AA)$ in the scattering range $(2 \theta)$ of $10-70^{\circ}$ with a scan rate of $0.02^{\circ} \mathrm{s}^{-1}$ and slit width of $0.1 \mathrm{~mm}$. Fourier transform infrared (FTIR) spectra were recorded on a Nicolet 5700 in transmission mode and with a wavenumber range of 400-4000 $\mathrm{cm}^{-1}$. A spectroscopic grade $\mathrm{KBr}$ disc was used for collecting the spectra with a resolution of $4 \mathrm{~cm}^{-1}$ performing 32 scans. Room temperature conductivity was measured via the four-probe method. Four contacts were made on the compressed pellet of the composite samples using conducting silver paste. These contacts were connected to a Keithley programmable current source (model 6221) and nanovoltmeter (model 2182A), and the conductivity was calculated based on Ohm's law. A thermogravimetric analyzer (Mettler Toledo TGA/SDTA 851e) was used to measure the thermal stability of the samples under an inert atmosphere (flowing $\mathrm{N}_{2}$ gas) in the temperature range of $25-900{ }^{\circ} \mathrm{C}$. The magnetic measurements were performed using a vibrating sample magnetometer (VSM) (model 7304 Lakeshore cryotronics Inc. USA) with a maximum magnetic field of $5 \mathrm{~T}$ and vibrating frequency of $76 \mathrm{~Hz}$. Electromagnetic shielding and dielectric measurements were carried out on an Agilent E8362B Vector network analyzer in the frequency range of 12.4$18 \mathrm{GHz}$ (Ku-band). Powder samples were compressed in the form of rectangular pellets $\left(15.8 \times 7.9 \times 1.5 \mathrm{~mm}^{3}\right)$, inserted into a copper sample holder and connected between the waveguide flanges of the network analyzer.

\section{Results and discussion}

\subsection{X-ray diffraction analysis}

Fig. 2a shows the XRD pattern of RGO, as well as the three different iron oxide structures and their respective composites with RGO. For the iron oxide nanorods, characteristic diffraction peaks were observed at $2 \theta=24.075^{\circ}(d=3.69355 \AA)$, $2 \theta=33.150^{\circ}(d=2.70022 \AA), 2 \theta=35.641^{\circ}(d=2.51703 \AA)$, $2 \theta=40.776^{\circ}(d=2.21114 \AA), 2 \theta=49.536^{\circ}(d=1.83868 \AA), 2 \theta=$ $53.981^{\circ}(d=1.69729 \AA), 2 \theta=57.372^{\circ}(d=1.60476 \AA), 2 \theta=$ $62.945^{\circ}(d=1.47543 \AA)$ and $2 \theta=64.204^{\circ}(d=1.44949 \AA)$, which correspond to the (012), (104), (110), (113), (024), (116), (018), (214) and (300) planes, respectively. The XRD pattern of the asprepared iron oxide nanorods matches with the standard structure (JCPDS card 33-0664), ${ }^{16}$ which corresponds to the $\alpha$-phase of iron oxide. It is interesting to note that the diffraction peaks at $2 \theta=30.18^{\circ}(d=2.959), 2 \theta=35.641^{\circ}$

Table 1 Details of the different parameters involved in the synthesis of different structures of iron oxide

\begin{tabular}{|c|c|c|c|c|c|c|c|c|}
\hline Nanorods & $\mathrm{FeSO}_{4} \cdot 7 \mathrm{H}_{2} \mathrm{O}$ & 14 & 35 & 8 & $\mathrm{CH}_{3} \mathrm{COONa} \cdot 3 \mathrm{H}_{2} \mathrm{O}$ & 13.6 & 12 & 120 \\
\hline Nanoflakes & $\mathrm{Fe}\left(\mathrm{NO}_{3}\right)_{3}$ & 41 & 100 & - & $\mathrm{NaOH}$ & $\begin{array}{l}10 \mathrm{M} \text { till } \\
\mathrm{pH} \geq 12\end{array}$ & 24 & 180 \\
\hline
\end{tabular}



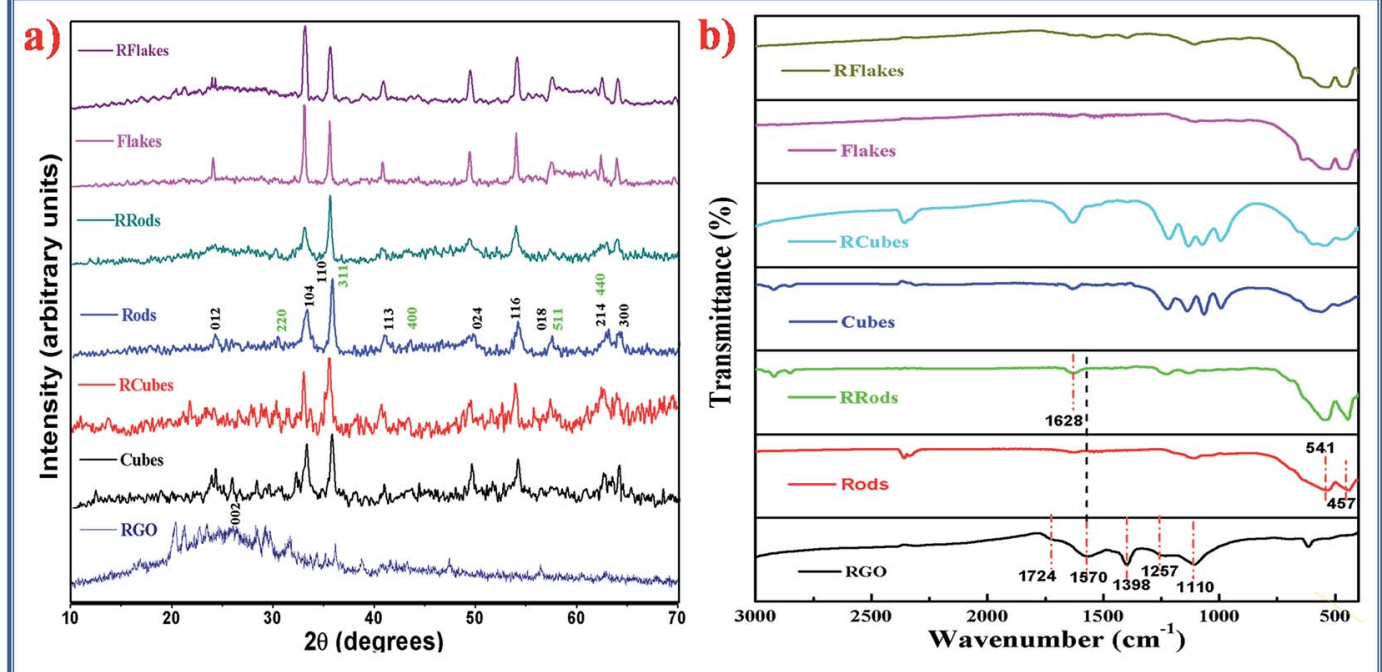

Fig. 2 (a) XRD of RGO, rods, RRods, cubes, RCubes, flakes and RFlakes and (b) a comparison of the FTIR spectra of RGO, rods, RRods, cubes, RCubes, flakes and RFlakes.

$(d=2.51703 \AA), 2 \theta=43.18^{\circ}(d=2.094), 2 \theta=57.372^{\circ}$ $(d=1.60476 \AA)$, and $2 \theta=62.945^{\circ}(d=1.47543 \AA)$ have also been observed in the XRD pattern of synthesized iron oxide. ${ }^{11}$ These peaks correspond to the (220), (311), (400), (511) and (440) planes of the $\gamma$-phase of iron oxide (matched with JCPDS card: 39-1346). Thus, it can be concluded that the as-prepared iron oxide has both phases of iron oxide, i.e. hematite $\left(\alpha-\mathrm{Fe}_{2} \mathrm{O}_{3}\right)$ and maghemite $\left(\gamma-\mathrm{Fe}_{2} \mathrm{O}_{3}\right)$ present. This can be justified by the fact that the phase transformation of iron oxide from $\gamma-\mathrm{Fe}_{2} \mathrm{O}_{3}$ to $\alpha$ $\mathrm{Fe}_{2} \mathrm{O}_{3}$ starts at $500{ }^{\circ} \mathrm{C}$ and is completed at $850{ }^{\circ} \mathrm{C}$ (ref. 17). As described in the synthesis part, during the preparation of the different iron oxide structures, the precipitate was obtained after hydrothermal treatment at above $600^{\circ} \mathrm{C}$ to obtain the iron oxide. This justifies the presence of both the $\alpha \& \gamma$ phase in the as-prepared iron oxide. Similarly, the main diffraction peaks for the iron oxide microcubes and iron oxide nanoflakes were observed at almost the same $2 \theta$ and with approximately similar interplanar distances. The reduction of GO was confirmed by its weak and broad diffraction peak at $2 \theta=25.53^{\circ},{ }^{18}$ which describes its typical amorphous carbon nature. In the case of the RGO composites, all the characteristic peaks of iron oxide can be clearly seen and the presence of the broad peak of RGO at $2 \theta=25.53^{\circ}$ can also be observed, as it suppresses the characteristic peak of iron oxide at $2 \theta=24.075^{\circ}$ to a great extent. ${ }^{19} \mathrm{All}$ of the characteristic peaks of the RGO composites show a slight shift in $2 \theta$ and decrease in their intensity in comparison to the peaks of pure iron oxide, which indicates the proper interaction between both of the compounds.

\subsection{Fourier-transform infrared (FTIR) spectroscopy}

The FTIR spectra of all the iron oxide structures and RGO-iron oxide composites are shown in Fig. 2b. The observed bands in the range of $400 \mathrm{~cm}^{-1}$ and $700 \mathrm{~cm}^{-1}$ are assigned to the $\mathrm{Fe}-\mathrm{O}-$ Fe vibration mode of the iron oxide nanorods, and the peaks at 541 and $457 \mathrm{~cm}^{-1}$ may be due to the transverse absorption $\left(E_{\mathrm{u}}\right)$ of iron oxide. ${ }^{6}$ The iron oxide microcubes and nanoflakes showed almost same bands, but with a slight shift of the transverse absorption mode to a higher frequency for the iron oxide microcubes, whereas it shifted to a lower frequency for iron oxide nanoflakes with respect to the iron oxide nanorods. This confirms that the vibrational modes of the FTIR spectra are shape-dependent for hematite particles. ${ }^{20}$ The FTIR spectra of RGO show $\mathrm{C}=\mathrm{O}$ carbonyl stretching at $1724 \mathrm{~cm}^{-1}$, a peak at $1398 \mathrm{~cm}^{-1}$ that can be assigned to the tertiary $\mathrm{C}-\mathrm{OH}$ groups, alkoxy stretching at $1110 \mathrm{~cm}^{-1}$ and epoxy stretching at 1257 $\mathrm{cm}^{-1} .{ }^{21}$ However, the low intensities of the peaks of these oxygen functional groups confirm the reduction of the GO to RGO. The $\mathrm{C}=\mathrm{C}$ stretching at $1570 \mathrm{~cm}^{-1}$ further confirms the formation of $\mathrm{RGO}^{22}$ The $\mathrm{C}=\mathrm{O}$ carbonyl stretching peak at $1724 \mathrm{~cm}^{-1}$ on RGO shifts to $1628 \mathrm{~cm}^{-1}$ in the FTIR spectrum of the RGO coated iron oxide nanorod composite, which may be due to the coordination of the $\mathrm{C}=\mathrm{O}$ group of RGO with Fe on the iron oxide surface (the formation of a-COO- compound). ${ }^{23}$ This confirms the strong bonding of iron oxide and RGO.

\subsection{Microstructural analysis}

Fig. 3 shows the SEM images of the different morphological structures of the iron oxide and RGO composites. Fig. 3a shows the iron oxide nanorods prepared using the hydrothermal treatment for $12 \mathrm{~h}$ at $120^{\circ} \mathrm{C}$. When the hydrothermal treatment duration was increased to $14 \mathrm{~h}$ and the temperature was increased to $160{ }^{\circ} \mathrm{C}$, iron oxide microcubes were formed, as shown in Fig. 3b. The inset image shows the estimated dimensions of a magnified cube from Fig. 3b. The dimensions of the microcube are observed to be $\sim 20 \times 20 \times 20 \mu \mathrm{m}$. A magnified view of the upper surface of the iron oxide cube is shown in the ESI, Fig. S2. $\dagger$ By further increasing the duration and temperature of the hydrothermal treatment to $24 \mathrm{~h}$ and $180{ }^{\circ} \mathrm{C}$, respectively, iron oxide flakes are observed, as shown in Fig. 3c. Fig. 3d-f show SEM micrographs of the RRod, RCube 


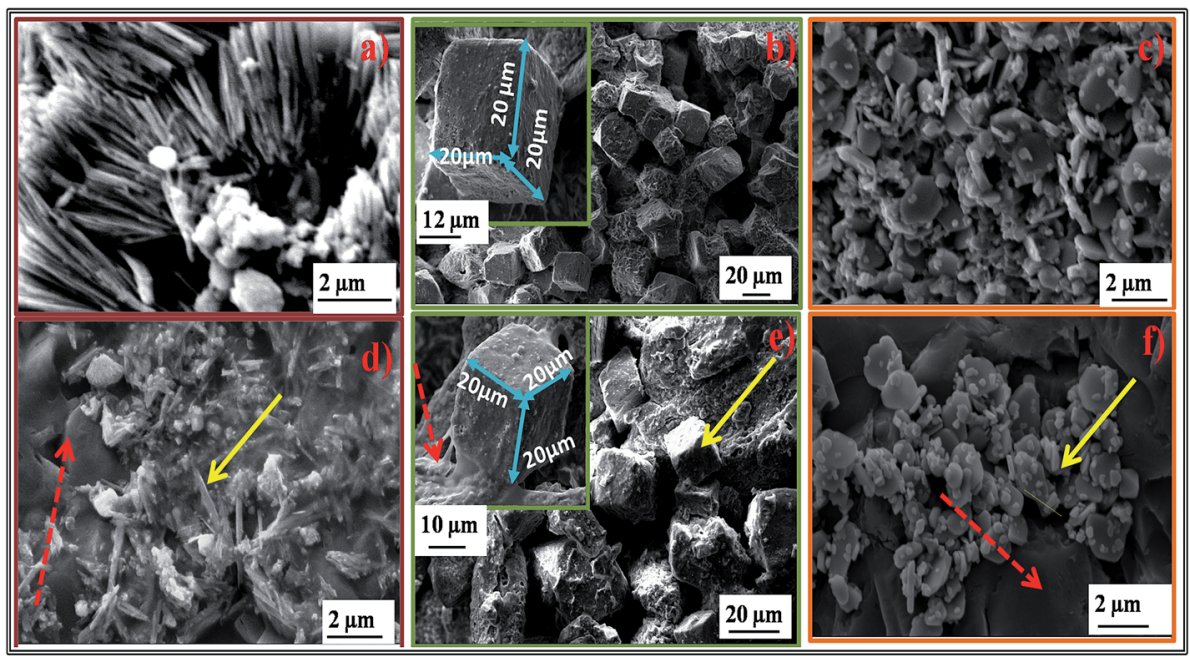

Fig. 3 SEM images of (a) iron oxide rods, (b) iron oxide cubes, (c) iron oxide flakes, (d) RRods, (e) RCubes and (f) RFlakes

and RFlake composites, respectively. These images show the effective covering of RGO sheets on the individual iron oxide shapes, which indicates the strong bonding and interaction of the different iron oxide shapes with the RGO sheets. In these images, the red arrow shows the RGO sheets, while the yellow arrow shows the different iron oxide shapes covered by RGO sheets.

TEM images of the different iron oxide shapes with distinct morphologies and RGO composites are shown in Fig. 4. The rod-like structure of the iron oxide particles was confirmed in Fig. 4a. Meanwhile, the inset of Fig. 4a shows a rod shaped iron oxide structure with an average length of $90 \mathrm{~nm}$ and average diameter of $10 \mathrm{~nm}$. Fig. 4b shows the TEM image of cubic iron oxide, which displays agglomeration between the cubic particles a few micrometers in size, which is also consistent with the SEM image. Fig. 4c displays the TEM image of iron oxide nanoflakes, while the inset shows the enlarged view of the lattice fringes. The average interplanar distance of the iron oxide nanoflakes was found to be $0.27 \mathrm{~nm}$, which corresponds to the (104) plane and is in good agreement with the XRD results of the iron oxide nanoflakes. It can easily be noticed from the typical HRTEM image of the iron oxide nanoflakes that the lattice fringes are clearly visible without any kind of distortion, which confirms the good crystallinity of the pristine iron oxide nanoflakes. The TEM image of the RGO composites shows the presence of RGO in the composite as thin areas, indicated using red arrows, while the presence of graphene and the iron oxide nanoparticles of different morphologies are shown as darker spots, indicated using yellow arrows in Fig. 4d-f. Furthermore, typical HRTEM images are shown in the insets of Fig. $4 \mathrm{~d}$ and $\mathrm{f}$, in which we selectively indicate the lattice fringes (encircled areas) of graphene with an estimated $d$ spacing $\sim 0.34 \mathrm{~nm}$.

\subsection{X-ray photoelectron spectroscopy}

XPS is a surface-sensitive quantitative spectroscopic technique that measures the elemental composition (in the parts per thousand range), empirical formula, chemical state and electronic state of the elements that exist within a material. In order to understand the surface interactions of the rod shaped iron

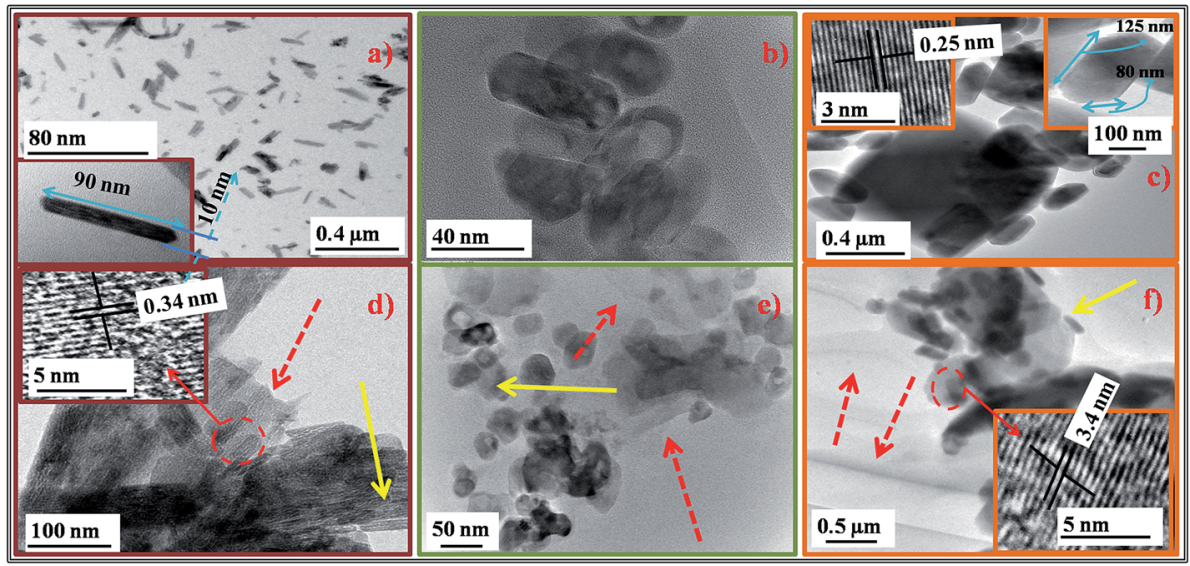

Fig. 4 TEM images of (a) iron oxide rods, (b) iron oxide cubes, (c) iron oxide flakes, (d) RRods, (e) RCubes and (f) RFlakes. 
oxide and RGO composite material, we employed the XPS technique for GO, RGO and the $\mathrm{Fe}_{2} \mathrm{O}_{3}-\mathrm{RGO}$ composite. Fig. 5 and 6 present the XPS spectra of GO, RGO and RGO- $\mathrm{Fe}_{2} \mathrm{O}_{3}$ rods, respectively. Fig. 5a shows the survey scan spectrum of GO and the inset shows the core level spectrum of $\mathrm{C} 1 \mathrm{~s}$. Fig. 5b shows the deconvoluted spectra of $\mathrm{C} 1 \mathrm{~s}$, where the presence of $\mathrm{C}-\mathrm{C}$, $\mathrm{C}=\mathrm{C}, \mathrm{C}-\mathrm{O}$ and $\mathrm{O}-\mathrm{C}=\mathrm{O}$ bonds can clearly be seen, which confirms the quality of the GO. Fig. 5c shows the survey scan spectrum of RGO and the inset shows the core level spectrum of C 1s. Fig. 5d presents the deconvoluted spectra of $\mathrm{C} 1 \mathrm{~s}$, where the presence of $\mathrm{C}-\mathrm{C}, \mathrm{C}=\mathrm{C}$ and $\mathrm{C}-\mathrm{O}$ can clearly be seen, which confirms the quality of the RGO. It can also be noticed from Fig. $5 \mathrm{a}$ and $\mathrm{c}$ that the ratio of $\mathrm{C} 1 \mathrm{~s}$ to $\mathrm{O} 1 \mathrm{~s}$ has been increased significantly in RGO in comparison to GO. The reduction of the functional groups can be easily seen from Fig. $5 \mathrm{~d}$, which is evidence of the complete reduction of GO to RGO. Furthermore, Fig. 6 shows the survey scan spectrum of $\mathrm{Fe}_{2} \mathrm{O}_{3}-\mathrm{RGO}$ and the inset shows the core level spectrum of $\mathrm{Fe} 2 \mathrm{p}_{3 / 2}$ and $\mathrm{Fe} 2 \mathrm{p}_{1 / 2}$. This clearly confirms the presence of iron oxide in the $\mathrm{Fe}_{2} \mathrm{O}_{3}-\mathrm{RGO}$ composite. It can also be seen from Fig. 6 that the intensities of $\mathrm{C} 1 \mathrm{~s}$ and $\mathrm{O}$ 1s are comparable. This may be because of the presence of oxygen in iron oxide. The obtained results are in good agreement with previously reported results on iron oxideRGO composites. ${ }^{24}$

\subsection{Magnetic properties}

We have also investigated the magnetic properties of the nanostructures based on the morphologies of the iron oxide particles and nanocomposites of iron oxide with RGO using a vibrating sample magnetometer (VSM). The magnetic

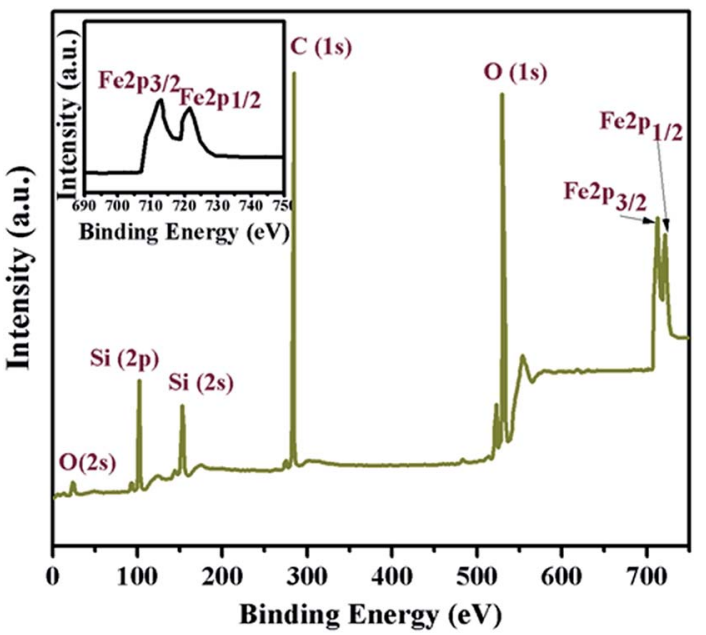

Fig. 6 (a) Survey scan spectrum of $\mathrm{RGO}-\mathrm{Fe}_{2} \mathrm{O}_{3}$ and the inset shows the core level spectrum of iron $p_{3 / 2}$ and $p_{1 / 2}$

properties of the different ferrite structures have been explained using an $M-H$ curve (Fig. 7a). The saturation magnetization $\left(M_{\mathrm{s}}\right)$ values of the ferrite nanorods, microcubes and nanoflakes were found to be $12.15 \mathrm{emu} \mathrm{g}^{-1}, 6.02 \mathrm{emu} \mathrm{g}^{-1}$ and $3.36 \mathrm{emu} \mathrm{g}^{-1}$, respectively, with an external field of 5 kOe having a small value of coercivity and negligible retentivity with a narrow hysteresis loop, indicating their superparamagnetic nature. ${ }^{25}$ It is well established in previous reports ${ }^{26-28}$ that the variation in the $M_{\mathrm{S}}$ value of iron oxide with various shapes may be caused by interparticle exchange interactions, their finite size and surface effects. The low $M_{\mathrm{s}}$ values between 3-12 emu $\mathrm{g}^{-1}$ can be

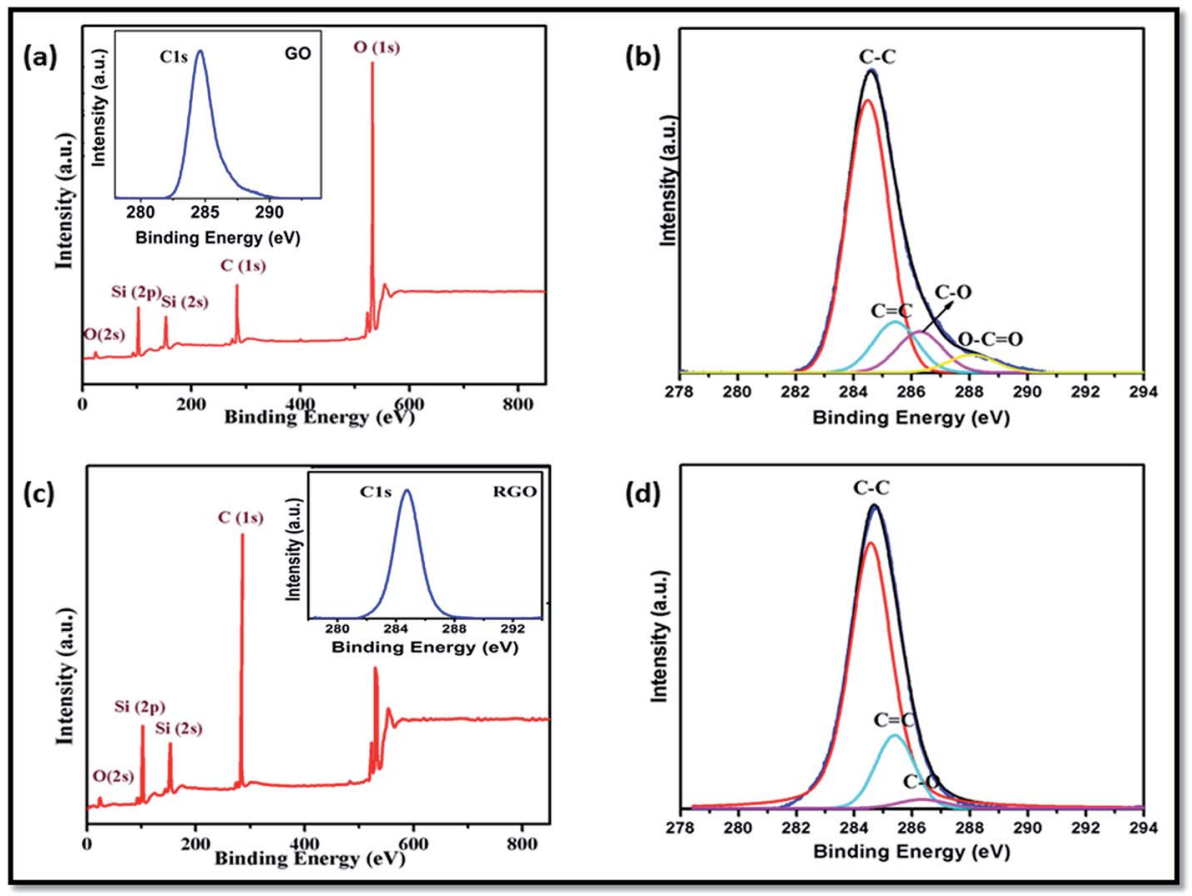

Fig. 5 (a) Survey scan spectrum of GO and the inset shows the core level spectrum of $C 1$ s, (b) deconvoluted spectra of $C$ 1s of GO, (c) survey scan spectrum of RGO and the inset shows the core level spectrum of $C 1$ s, and (d) deconvoluted spectra of $C 1 s$ of RGO. 

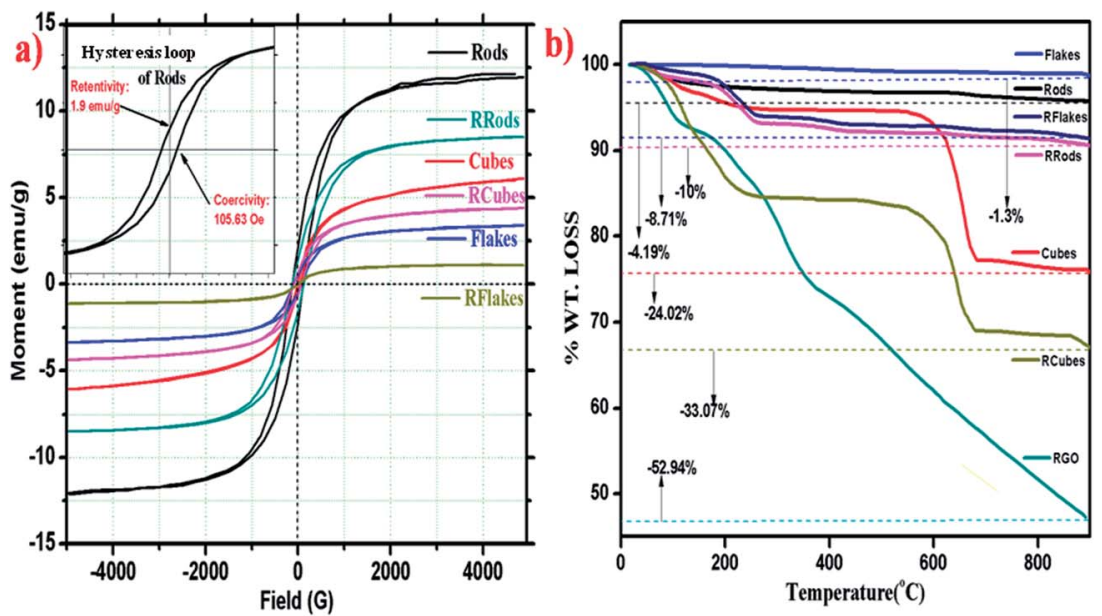

Fig. 7 (a) Vibrating sample magnetometer plots of the rods, RRods, cubes, RCubes, flakes and RFlakes. (b) TGA curves of the RGO, rods, RRods, cubes, RCubes, flakes and RFlakes.

explained by the fact that surface modification usually leads to a decrease in the $M_{\mathrm{s}}$ value, on account of the possible damage to the magnetic cores or increase in the coating contents. The low $M_{\mathrm{S}}$ value can also be justified by both the hematite and maghemite phase being present in the as-prepared iron oxide, and so the magnetization values of the samples are not as low as would generally be found in $\alpha-\mathrm{Fe}_{2} \mathrm{O}_{3}$ and not as outstanding as would normally be seen in $\gamma-\mathrm{Fe}_{2} \mathrm{O}_{3}$. But the $M_{\mathrm{s}}$ values between 3-12 emu g ${ }^{-1}$ are significant enough to increase the absorption loss for electromagnetic shielding. The significant rise in the $M_{\mathrm{s}}$ value of the rod structures clearly indicates the merits of their linear structures. ${ }^{16}$ When the different ferrite nanostructures were encapsulated in RGO, the $M_{\mathrm{s}}$ values of the RRods, RCubes, and RFlakes were decreased to $8.48 \mathrm{emu} \mathrm{g}^{-1}, 4.37 \mathrm{emu} \mathrm{g}^{-1}$ and $1.11 \mathrm{emu}^{-1}$, respectively, at an external field of $5 \mathrm{kOe}$. In these cases, a small coercivity and negligible retentivity were also observed.

\subsection{Thermal stability}

The thermal stability of the different iron oxide nanostructures, RGO and their composites has been studied using thermogravimetric analysis. The pure nanorods, microcubes and nanoflakes of iron oxide show excellent thermal stability, with weight losses of $4 \%, 24 \%$ and $0.5 \%$, respectively, up to $900{ }^{\circ} \mathrm{C}$, as shown in Fig. 7b. On the other hand, RGO shows a two-step weight loss, with the first step corresponding to a weight loss of around $-15 \%$ over the temperature range of $25-230{ }^{\circ} \mathrm{C}$, which may be due to the presence of moisture, residual solvent and unstable oxygen functional groups. ${ }^{23}$ The second stage of thermal degradation in RGO is found over the temperature range of $350{ }^{\circ} \mathrm{C}-900{ }^{\circ} \mathrm{C}$ with a weight loss of $28 \%$, which corresponds to the presence of stable oxygen functional groups. RGO has an overall weight loss of $52.94 \%$ up to $900{ }^{\circ} \mathrm{C}$. Combining RGO with iron oxide decreases the thermal stability of the iron oxide to a considerable extent. The RRod, RCube and RFlake composites showed the weight losses of 8.71\%, 33.07\% and $4.19 \%$, respectively, up to $900{ }^{\circ} \mathrm{C}$. RGO has a very high weight loss $(16.5 \%)$ in the temperature range of $134{ }^{\circ} \mathrm{C}-343{ }^{\circ} \mathrm{C}$, and the iron oxide-RGO composites showed their maximum weight loss in this range; with $3.5 \%$ weight loss in the $201{ }^{\circ} \mathrm{C}-$ $254{ }^{\circ} \mathrm{C}$ range for RRods, $3 \%$ weight loss in the $200{ }^{\circ} \mathrm{C}-257^{\circ} \mathrm{C}$ range for RFlakes and $11 \%$ weight loss in the $102{ }^{\circ} \mathrm{C}-250{ }^{\circ} \mathrm{C}$ range for RCubes. It is interesting to note that the iron oxideRGO composites have a highly stable thermal phase in the $350{ }^{\circ} \mathrm{C}-900{ }^{\circ} \mathrm{C}$ range in comparison to the RGO, which has a gradual weight loss in this range. From this, it can be concluded that the iron oxide and RGO form a strong interaction, which results in the eradication of stable oxygen functional groups like -COO- in the iron oxide-RGO composite.

\subsection{Raman spectroscopy}

The structural changes occurring after the encapsulation of iron oxide in RGO has been studied using Raman spectroscopy. Raman spectra of all the iron oxide nanostructures and their composites with RGO are shown in Fig. 8. All the prominent peaks of iron oxide at $296.18,405,501,611$ and $1315 \mathrm{~cm}^{-1}$ are clearly visible for the iron oxide nanorods. ${ }^{29}$ Iron oxide microcubes and nanoflakes also have their hematite phase peaks around only these values. In the Raman spectrum of the iron oxide nanorods-RGO composite, both the D and G band peaks of RGO are clearly visible with a slight red shift. This may be due to the overlapping of the D band peak with the hematite phase peak of iron oxide at $1315 \mathrm{~cm}^{-1}$ (ref. 18) and the suppressed iron oxide phase peaks are also clearly visible in the spectrum of the composite (see ESI Fig. S3 and Table S1 $\dagger$ ). The $I_{\mathrm{D}} / I_{\mathrm{G}}$ value of iron oxide-RGO is higher (1.66 for rods, 1.19 for cubes, 1.23 for flakes) than that of RGO (1.00). This also suggests the interaction of $\mathrm{Fe}_{2} \mathrm{O}_{3}$ with the $\mathrm{RGO}$ sheet, because iron oxide interacted with the available defect sites of the RGO sheet, or with further occurrences of defect sites, at the time of the formation of the $\mathrm{Fe}_{2} \mathrm{O}_{3}$-RGO composites. ${ }^{30}$ It is interesting to note that the Raman spectra show the D/G intensity ratios to be more than 1 in all three iron oxide-RGO composites and also higher than 


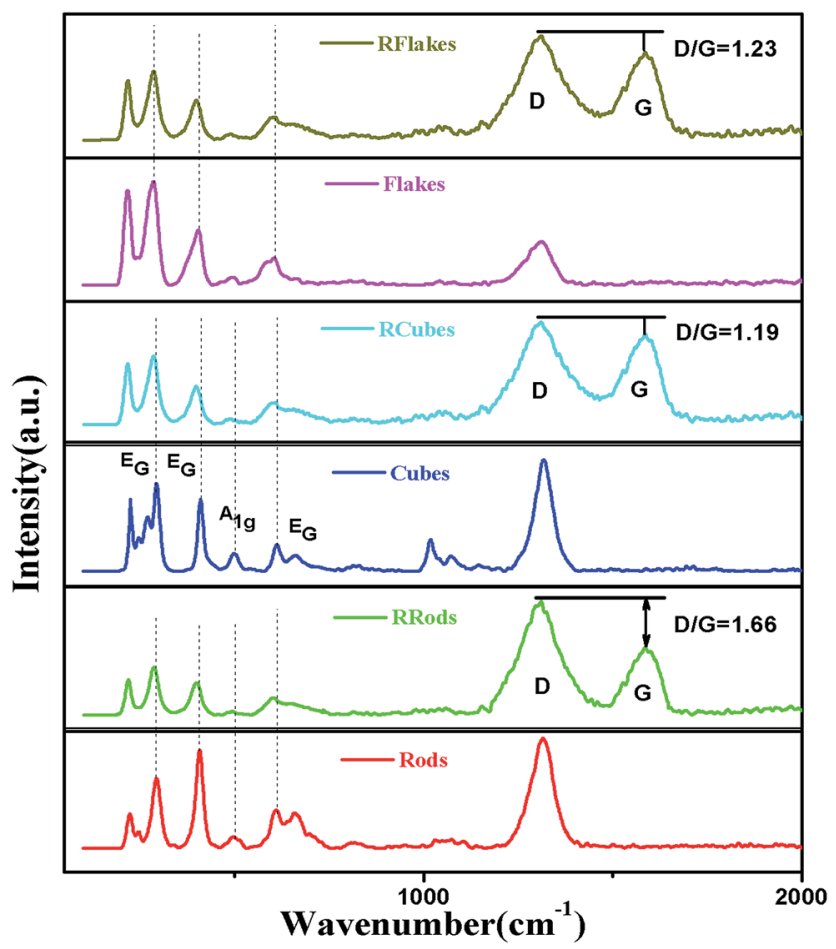

Fig. 8 Raman spectra of the rods, RRods, cubes, RCubes, flakes and RFlakes.

that of pristine RGO, which confirms the further reduction of RGO in the composite. ${ }^{31}$

\subsection{Shielding measurements}

The EMI shielding effectiveness (SE) of a material is explained in terms of the ratio of the incident and transmitted energy and can be represented mathematically on the logarithmic scale as $\mathrm{SE}_{\mathrm{T}}(\mathrm{dB})=-10 \log \left\{P_{\mathrm{T}} / P_{\mathrm{I}}\right\}$, where $P_{\mathrm{I}}$ and $P_{\mathrm{T}}$ are the power of the incident and transmitted electromagnetic waves, respectively. A material's total shielding effectiveness $\left(\mathrm{SE}_{\mathrm{T}}\right)$ is a contribution of three components, viz. absorption $\left(\mathrm{SE}_{\mathrm{A}}\right)$, reflection $\left(\mathrm{SE}_{\mathrm{R}}\right)$ and multiple internal reflection $\left(\mathrm{SE}_{\mathrm{M}}\right)$. The reflection $(R)$, transmission $(T)$ and absorption $(A)$ components were obtained through the measurement of scattering parameters S11 (or S22) and S21 (or S12) of a two port network analyzer, where $R=$ $|\mathrm{S} 11|^{2}$ and $T=|\mathrm{S} 21|^{2}$ and $A=1-|\mathrm{S} 11|^{2}-|\mathrm{S} 21|^{2}$. The primary mechanism for EMI shielding is reflection, for which the shield possesses mobile charge carriers that can interact with electromagnetic waves and create ohmic loss in the shield. Thus, the shield needs moderate electrical conductivity of around $10^{-3}$ to $1 \mathrm{~S} \mathrm{~m}^{-5}$. The strong and effective secondary mechanism is absorption, for which the shield should have electric/ magnetic dipoles that can interact with electromagnetic radiation. Fig. 9 shows the dependence of the shielding effectiveness in the 8.2-12.4 GHz range. The experimental measurements reveal that the shielding effectiveness values for flakes, cubes and rods are found to be $2.83,4.27$ and 5.40, respectively. When these structures are covered with RGO, the SE values of RFlakes RCubes and RRods are found to be 27.42, 30.96 and 33.30,

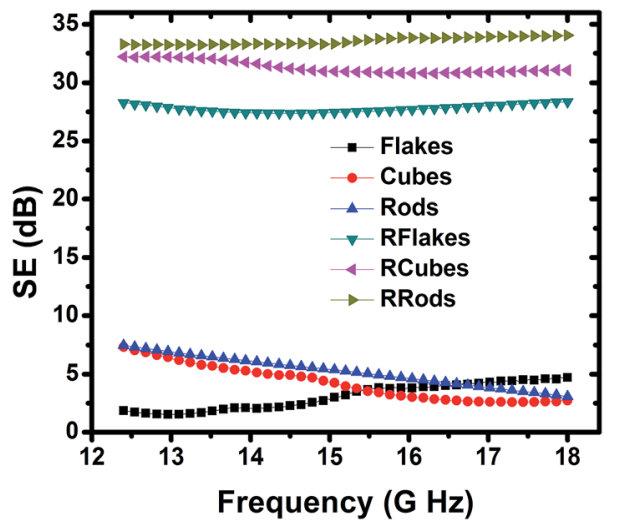

Fig. 9 Variation of the total shielding effectiveness with frequency.

respectively. These results are associated with the shape and size of iron oxide, as shown in Fig. 9. Thus the maximum total $\mathrm{SE}$ achieved for the RRods, due to the improved microwave shielding properties of the RRods, may be ascribed to the rod shaped nature of iron oxide. The results suggest that the systems are promising EMI absorption materials in the microwave frequency range. In addition, the enhanced microwave shielding properties of the $\mathrm{Fe}_{3} \mathrm{O}_{4}$ microstructures may be due to the specific rod-shaped structures. The assembly of these rods is believed to increase the geometrical effect and lead to enhanced shielding effectiveness with the incidence of microwaves. Furthermore, RRods offer a higher aspect ratio in comparison to the RCubes and RFlakes. The rod shaped nature of iron oxide also allows it to be aligned in a specific direction to maximize the attenuation of an EM wave, while the cubes and flakes are randomly scattered, and are not able to maximize the attenuation of microwaves. ${ }^{32}$ The plausible proposed mechanism for the enhancement of the EMI shielding by the nanorod shape in comparison to the other structures is shown in Fig. 10. In other words, one-dimensional nanostructures with a tremendous effective surface area (due to high aspect ratio) appear to be good candidates for microwave shielding. ${ }^{33-35}$

In all of the composite materials, the observed attenuation is the result of magnetic and dielectric loss (see the ESI $\dagger$ for the dielectric parameters of RRods, Fig. S4 $\dagger$ ). Magnetic loss comes from eddy current effects, natural resonance and anisotropy energy present in the composites. In the microwave range, the presence of nanoferrite particles in the composite is the main cause of the eddy current. The anisotropy energy of the small size materials would be higher due to the surface anisotropy field, as a result of the small size effect. The higher anisotropy energy also contributes to the enhancement of microwave absorption. In addition, the coating of the magnetic iron oxide with an RGO layer introduces additional interfaces and more polarization charges at the surface. ${ }^{31}$ Interfacial polarization is an important polarization process and the associated relaxation will also give rise to a loss mechanism. It is reasonable to expect that the dielectric loss may be due to the significant contributions of interfacial polarization. ${ }^{36}$ It is well known that 

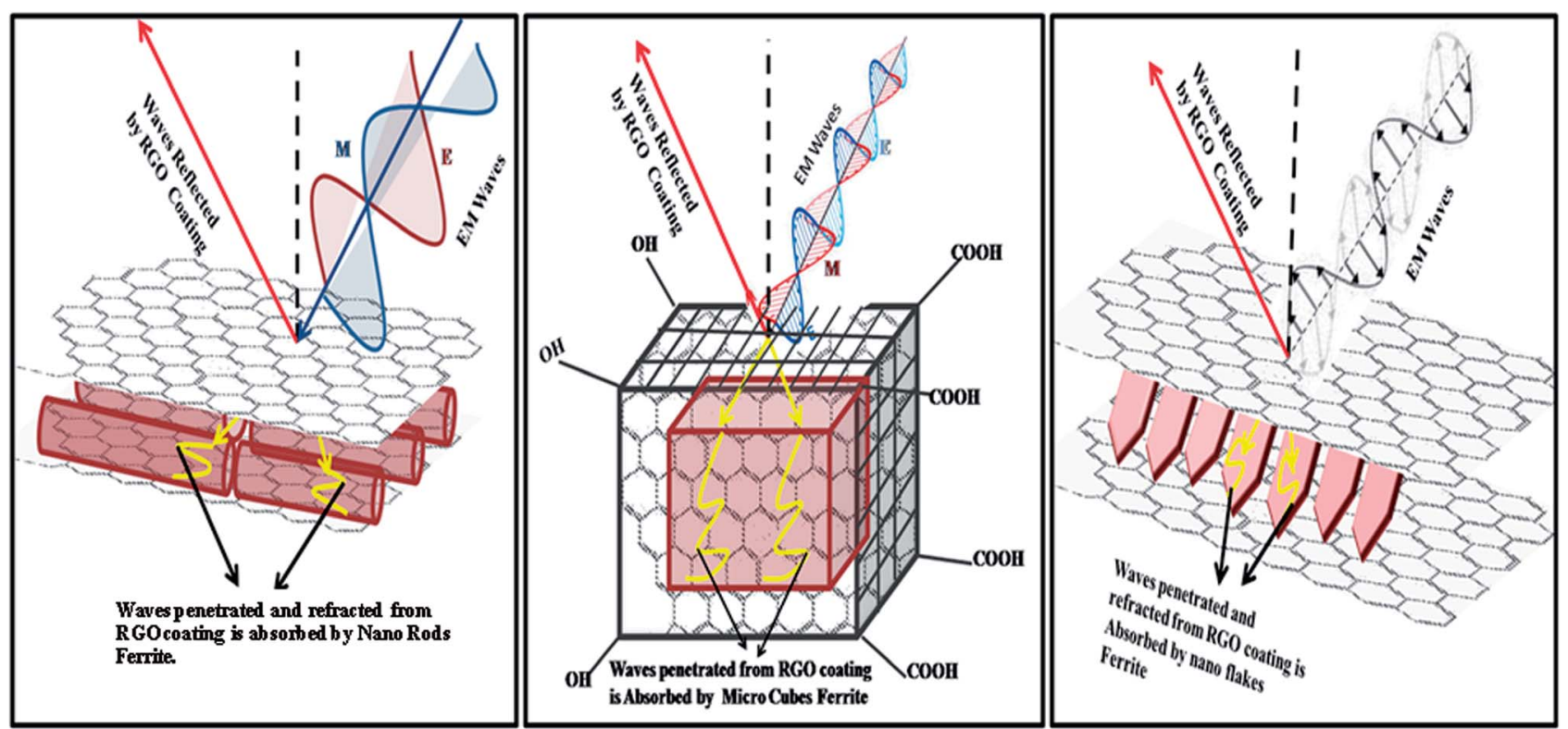

Fig. 10 Schematic representation of the interaction of microwave with different shape RGO coated iron oxide.

molecular dipoles formed at the surface interact with the microwave field, leading to some absorption losses through heating.

Furthermore, the sufficient states induced by defects and residual bonds on the extremely thin and highly polar RGO sheets increase the absorption of the microwave. ${ }^{37,38}$ With the high mass ratio of the RGO, conductivity plays the main role in EM wave attenuation..$^{31}$ The conductivity of the RGO is beneficial for energy attenuation, and increasing the conductivity would increase the energy conversion effectiveness of the composites. Therefore, the results shown in this work indicate the fundamental principles for achieving high-performance EMI shielding materials.

\section{Conclusions}

We have successfully synthesized various morphologies of iron oxide with controlled shapes and sizes. We have also demonstrated that all of the shapes of iron oxide covered with RGO exhibit promising EMI shielding performance. The iron oxide nanorods covered with RGO show higher EMI shielding values than the composites based on the other structures. The presence of RGO plays a crucial role in enhancing the dielectric loss. The enhanced dielectric loss can be attributed to the natural resonance, dipole relaxation, electron polarization related relaxation, interfacial polarization, residual defects in thinner RGO sheets and higher conductivity of the composite. Additionally, the associated magnetic losses of the composites are mainly caused by natural resonance and eddy currents. Thus, these new composites provide new insights into EMI shielding materials and may represent suitable morphologies for applications in next generation EMI shielding applications.

\section{Acknowledgements}

The authors wish to thank Prof. R. C. Budhani, Director, N.P.L., for his keen interest in the work. The authors also thank Dr N. Vijayan and K. N. Sood for recording the XRD patterns and SEM micrographs, respectively.

\section{References}

1 Z. Chen, C. Xu, C. Ma, W. Ren and H.-M. Cheng, Adv. Mater, 2013, 25, 1296-1300.

2 T. K. Gupta, B. P. Singh, V. N. Singh, S. Teotia, A. P. Singh, I. Elizabeth, S. R. Dhakate, S. K. Dhawan and R. B. Mathur, J. Mater. Chem. A, 2013, 2, 4256-4263.

3 Y. Yang, M. C. Gupta, K. L. Dudley and R. W. Lawrence, Nano Lett., 2005, 5, 2131-2134.

4 A. P. Singh, M. Mishra, P. Sambyal, B. K. Gupta, B. P. Singh, A. Chandra and S. K. Dhawan, J. Mater. Chem. A, 2014, 2, 3581-3593.

5 A. P. Singh, B. K. Gupta, M. Mishra, G. Govind, A. Chandra, R. B. Mathur and S. K. Dhawan, Carbon, 2013, 56, 86-96.

6 Y. M. Zhao, Y. H. Li, R. Z. Ma, M. J. Roe, D. G. McCartney and Y. Q. Zhu, Small, 2006, 2, 422-427.

7 S. Zeng, K. Tang and T. Li, J. Colloid Interface Sci., 2007, 312, 513-521.

8 I. T. Kim, A. Magasinski, K. Jacob, G. Yushin and R. Tannenbaum, Carbon, 2013, 52, 56-64.

9 A. P. Singh, M. Mishra, A. Chandra and S. Dhawan, Nanotechnology, 2011, 22, 465701.

10 A. P. Singh, P. Garg, F. Alam, K. Singh, R. Mathur, R. Tandon, A. Chandra and S. Dhawan, Carbon, 2012, 50, 3868-3875.

11 V. Chandra, J. Park, Y. Chun, J. W. Lee, I.-C. Hwang and K. S. Kim, ACS Nano, 2010, 4, 3979-3986. 
12 A. Celzard, E. McRae, G. Furdin and J. F. Marêché, J. Phys.: Condens. Matter, 1997, 9, 2225.

13 B. Wen, X. X. Wang, W. Q. Cao, H. L. Shi, M. M. Lu, G. Wang, H. B. Jin, W. Z. Wang, J. Yuan and M. S. Cao, Nanoscale, 2014, 6, 5754-5761.

14 S. Zeng, K. Tang and T. Li, J. Colloid Interface Sci., 2007, 312, 513-521.

15 B. Tang, G. Wang, L. Zhuo, J. Ge and L. Cui, Inorg. Chem., 2006, 45, 5196-5200.

16 N. K. Chaudhari and J.-S. Yu, J. Phys. Chem. C, 2008, 112, 19957-19962.

17 S. Sahoo, K. Agarwal, A. Singh, B. Polke and K. Raha, Int. J. Eng. Sci. Tech., 2010, 2, 118-126.

18 H. Zhang, A. Xie, C. Wang, H. Wang, Y. Shen and X. Tian, J. Mater. Chem. A, 2013, 1, 8547-8552.

19 M. Du, C. Xu, J. Sun and L. Gao, J. Mater. Chem. A, 2013, 1, 7154-7158.

20 R. Ramesh, K. Ashok, G. Bhalero, S. Ponnusamy and C. Muthamizhchelvan, Cryst. Res. Technol., 2010, 45, 965968.

21 M. Zhou, Y. Zhai and S. Dong, Anal. Chem., 2009, 81, 56035613.

22 V. Loryuenyong, K. Totepvimarn, P. Eimburanapravat, W. Boonchompoo and A. Buasri, Adv. Mater. Sci. Eng., 2013, 2013, 923403.

23 X. Yang, X. Zhang, Y. Ma, Y. Huang, Y. Wang and Y. Chen, J. Mater. Chem., 2009, 19, 2710-2714.

24 X. Yang, C. Chen, J. Li, G. Zhao, X. Ren and X. Wang, RSC Adv., 2012, 2, 8821-8826.
25 M. Mishra, A. P. Singh and S. Dhawan, J. Alloys Compd., 2013, 557, 244-251.

26 H.-L. Xu, H. Bi and R.-B. Yang, J. Appl. Phys., 2012, 111, 07A522.

27 Z. Wang, J. Zou, Z. Ding, J. Wu, P. Wang, S. Jin and H. Bi, Mater. Chem. Phys., 2013, 142, 119-123.

28 B. Philip, J. Xie, A. Chandrasekhar, J. Abraham and V. K. Varadan, Smart Mater. Struct., 2004, 13, 295.

29 H.-J. Song, X.-H. Jia, N. Li, X.-F. Yang and H. Tang, J. Mater. Chem., 2012, 22, 895-902.

30 M. Mishra, A. P. Singh, B. P. Singh and S. K. Dhawan, RSC Adv., 2014, 4, 25904-25911.

31 M. Mishra, A. P. Singh, B. P. Singh, V. N. Singh and S. K. Dhawan, J. Mater. Chem. A, 2014, 2, 13159-13168.

32 A. Srivastava, A. K. Srivastava and O. N. Srivastava, Carbon, 2001, 39, 201-206.

33 P. Ajayan, Chem. Rev., 1999, 99, 1787-1800.

34 P. M. Ajayan, Nature, 1993, 361, 333-334.

35 H. T. Feng, R. F. Zhuo, J. T. Chen, D. Yan, J. J. Feng, H. J. Li, S. Cheng, Z. G. Wu, J. Wang and P. X. Yan, Nanoscale Res. Lett., 2009, 4, 1452-1457.

36 H. Li, Y. Huang, G. Sun, X. Yan, Y. Yang, J. Wang and Y. Zhang, J. Phys. Chem. C, 2010, 114, 10088-10091.

37 B. Wen, X. X. Wang, W. Q. Cao, H. L. Shi, M. M. Lu, G. Wang, H. B. Jin, W. Z. Wang, J. Yuan and M. S. Cao, Nanoscale, 2014, 5454-5461.

38 P. C. P. Watts, W. K. Hsu, A. Barnes and B. Chambers, Adv. Mater., 2003, 15, 600-603. 